\title{
Business \& Information Systems Engineering (BISE) ist AIS Affiliated Journal
}

\section{DOI 10.1007/s11576-010-0259-6}

\section{Der Autor}

Prof. Dr. Hans Ulrich Buhl ( $₫)$

FIM Kernkompetenzzentrum Finanz\& Informationsmanagement

Universität Augsburg

Universitätsstraße 12

86159 Augsburg

Deutschland

hans-ulrich.buhl@

wiwi.uni-augsburg.de

Online publiziert: 2011-01-04

This article is also available in English via http://www.springerlink.com and http://www.bise-journal.org: Buhl HU (2010) Business \& Information Systems Engineering (BISE) is AIS Affiliated Journal. Bus Inf Syst Eng. doi: 10.1007/s12599-010-0144-2.

(C) Gabler Verlag 2010
In Heft 4/2010 konnte ich Ihnen die ersten Ergebnisse unserer Internationalisierungsstrategie, insbesondere den starken Zuwachs der Downloadzahlen unserer Zeitschrift vorstellen. Auch unser Impact Factor hat sich von 2007 auf 2009 mehr als verdoppelt, noch bevor sich die Internationalisierung der Zeitschrift auf diesen auswirkt. Wir sind also zuversichtlich, dass sich dieser Trend weiter fortsetzen wird.

Heute freue ich mich, Ihnen einen weiteren wichtigen Schritt in Richtung einer erfolgreichen Realisierung unserer Strategie mitteilen zu können:

Im Rahmen der ICIS 2010 wurde die BISE offiziell als AIS Affiliated Journal angekündigt. Damit möchte die Association for Information Systems (AIS) die BISE aufgrund ihres innovativen Ansatzes zur Verbesserung der Kommunikation zwischen der BISE- und ISCommunity unterstützen und fördern. Aus diesem Grund darf die BISE als erste
Zeitschrift dieser Art in Zukunft die Bezeichnung „AIS Affiliated Journal“ führen.

Im Rahmen der Affiliation ist die BISE künftig auch über die AIS E-Library (http://aisel.aisnet.org/bise) - und damit frei für alle Mitglieder der AIS - verfügbar. Damit ist es uns möglich, unsere internationale Sichtbarkeit noch weiter zu erhöhen.

Bei dieser Gelegenheit möchte ich Jan vom Brocke, Guy Fitzgerald, Kalle Lyytinen, Dov Te'eni, Pete Tinsley, Cathy Urquhart und Robert Winter für Ihre wertvolle Unterstützung danken. Ein ganz besonderes Dankeschön gilt Marco de Marco, der uns sehr stark unterstützt und bei der Erreichung dieses Ziels geholfen hat.

Prof. Dr. Hans Ulrich Buhl Geschäftsführender Herausgeber 